\title{
L'aménagement durable des vieilles forêts boréales : mythes, pistes de solutions et défis ${ }^{1}$
}

\author{
par Héloïse Le Goffe,3, Louis De Grandpré ${ }^{3}$, Daniel Kneeshaw² et Pierre Bernier ${ }^{3}$
}

\begin{abstract}
RÉSUMÉ
Les vieilles forêts boréales cristallisent plusieurs problématiques forestières comme l'aménagement forestier durable et le développement d'un réseau d’aires protégées, mais aussi la gestion adaptative et participative des ressources naturelles. Ainsi, les vieilles forêts boréales offrent une bonne occasion pour l'industrie forestière de développer une gestion transparente qui intègre lensemble des valeurs sociales associées aux vieilles forêts. Dans cet article, nous proposons une revue des enjeux actuels reliés à l’aménagement durable et à la conservation des vieilles forêts boréales sous la forme de mythes et de solutions. Pour terminer, nous identifions et nous discutons des limites de notre compréhension de ces enjeux et nous proposons des priorités de recherche pour y remédier.
\end{abstract}

Mots clés : aménagement forestier durable, vieilles forêts boréales, biodiversité, produits forestiers, valeurs sociales, systèmes sylvicoles adaptés

\section{ABSTRACT}

Old-growth boreal forests serve as focal points for many issues affecting the forest sector such as sustainable forest management and the development of a conservation network. They also challenge the implementation of an adaptive management framework and participative natural resources management. Old-growth boreal forests thus provide a good opportunity for the forest sector to develop transparent management that integrates the diversity of social values associated with old-growth boreal forests. In this paper, we present a review of the different issues related to the sustainable management and conservation of old-growth boreal forests and present these issues in terms of myths and solutions. Finally, we identify and discuss the current limits of our understanding of these issues and we propose research priorities to bridge these knowledge gaps.

Key words: sustainable forest management, old-growth boreal forests, biodiversity, social values, adapted silvicultural systems

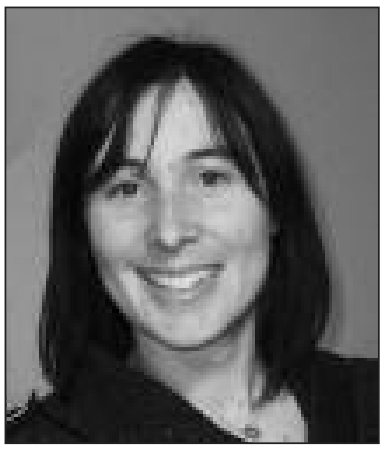

Héloïse Le Goff

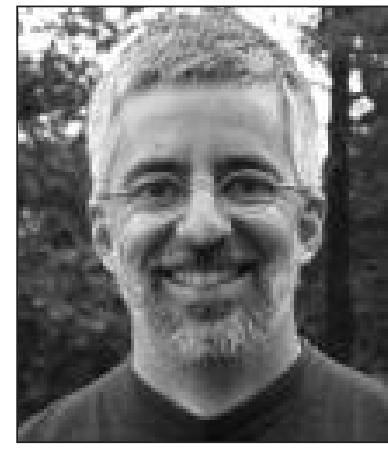

Louis De Grandpré

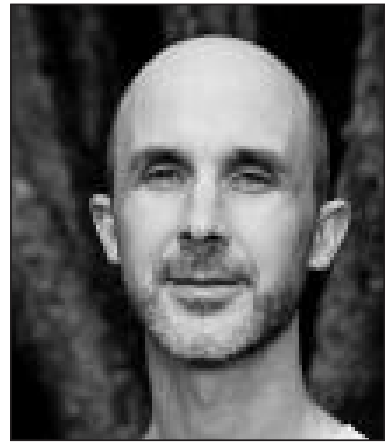

Daniel Kneeshaw

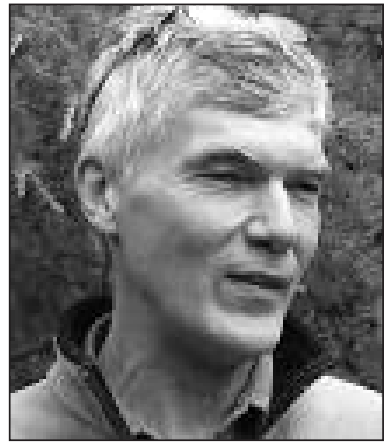

Pierre Bernier

\section{Introduction}

Dans le contexte actuel de la crise forestière, conserver davantage de vieilles forêts boréales est souvent interprété comme une diminution des volumes de bois acheminés aux usines. Pour ceux qui vivent de l'exploitation forestière, lenjeu des vieilles forêts apparaît souvent comme une charge supplémentaire qui aggrave la crise forestière. Pourtant, grâce à leur diversité structurale et fonctionnelle, les vieilles forêts boréales

pourraient faire partie des solutions à la crise forestière en encourageant une diversification des pratiques forestières et de l'économie locale des communautés forestières.

Le présent texte fait suite à un atelier sur les vieilles forêts boréales qui seest déroulé en mai 2008 à Sept-Îles, Québec. Le succès de latelier témoigne bien du besoin de mécanismes d'échange et d'interaction sur l'importance écologique des vieilles forêts et leur contribution économique et sociale. En

\footnotetext{
${ }^{1}$ Cet article s'appuie sur les présentations et les ateliers qui ont eu lieu lors du colloque « Les vieilles forêts boréales : leur place dans l'aménagement durable » tenu en mai 2008 à Sept-îles, Québec.

${ }^{2}$ Centre détude de la forêt, Université du Québec à Montréal, succ. C.P. 8888, Centre-Ville, Montréal, QC H3C 3P8. Courriel : heloise. legoff@nrcan.gc.ca.

${ }^{3}$ Service canadien des forêts, Centre de foresterie des Laurentides, 1055, rue du PEPS, C.P. 10380, succ. Sainte-Foy, Québec, QC G1V 4C7.
} 
effet, un cadre d'échange dynamique et continu des connaissances permet aux différentes parties concernées de partager leurs préoccupations et de faire ressortir des éléments de réponse en amont du processus décisionnel. Cette approche peut donc favoriser l'adoption de solutions plus efficaces et mieux acceptées par les différents intervenants (Bouthillier et Roberge 2007, Bouthillier 2009).

La principale différence observée entre un paysage forestier boréal naturel et un paysage forestier boréal aménagé de façon équienne est la raréfaction des vieilles forêts, leur répartition, ainsi que leur structure (Jetté et al. 2008). Alors que les vieilles forêts dominent les paysages forestiers préindustriels et non aménagés, l'aménagement équienne utilisé en forêt boréale aboutit à une raréfaction des vieux peuplements. Tel qu'il est pratiqué actuellement, l'aménagement forestier prélève des forêts matures et surannées sans prévoir de mesures particulières pour maintenir des massifs intacts ou des peuplements avec des attributs de vieilles forêts (débris ligneux, structure étagée et inéquienne) dans les zones aménagées. La raréfaction des massifs de forêt ayant dépassé le stade mature menace les espèces associées aux vieilles forêts boréales comme certaines mousses et certains lichens, mais aussi certains oiseaux et le caribou forestier (Boudreault 2001, Courtois et al. 2001, Euler et Wedeles 2005).

Malgré les préoccupations souvent soulevées concernant le maintien ou l'aménagement des vieilles forêts, nous disposons déjà d'une certaine compréhension de la structure et de la dynamique des vieilles forêts boréales qui nous permet de développer des stratégies d’aménagement et des options sylvicoles inéquiennes mieux adaptées. Dans ce texte, nous proposons une mise au point sur les principaux mythes associés à laménagement et à la conservation des vieilles forêts boréales à la lumière des connaissances récentes. Ensuite, nous présentons les pistes de solutions proposées par les différents intervenants lors des ateliers. Pour terminer, nous identifions les limites de notre compréhension des vieilles forêts boréales et nous proposons des priorités de recherche pour y remédier.

\section{Qu'est-ce qu'une vieille forêt boréale?}

À léchelle du peuplement, une vieille forêt boréale se caractérise par une structure complexe où se côtoient des arbres issus de plusieurs cohortes qui se sont suivies ou chevauchées depuis la perturbation d’origine. La mortalité s'y produit généralement à léchelle de l'arbre ou par petits groupes d’arbres, ce qui engendre une dynamique par trouées (McCarthy 2001, Pham et al. 2004, De Grandpré et al. 2008). Le stade de vieilles forêts commence au début de la mortalité de la cohorte dominante (canopée) initiée après la dernière perturbation sévère. À léchelle du paysage, les vieilles forêts sont dominées par les peuplements résineux dont la densité et la hauteur varient au cours du temps au gré des perturbations partielles et des épidémies d'insectes qui réinitialisent la succession forestière d'une partie des peuplements. Comme ces variations ne sont pas synchrones d'un peuplement à l'autre, la composition, la hauteur et la densité varient d'un peuplement à lautre, mais restent relativement stables à léchelle du paysage (Gauthier 2009).

\section{Les mythes associés aux vieilles forêts boréales Les vieilles forêts boréales dépérissent}

Le mythe le plus répandu sur les vieilles forêts boréales est que celles-ci sont souvent associées à un stade de dépérissement au cours duquel le volume de matière ligneuse sur pied diminue jusquà devenir null. L'interprétation des tables de production utilisées pour le calcul de la possibilité a largement contribué à cette idée car on y confond lâge des arbres et l'âge des peuplements (Pothier et Savard 1998). On attribue aux vieilles forêts la propriété de sénescence, une propriété qui ne se manifeste réellement quà léchelle de l’arbre. De plus, comme ces tables ont été développées pour des peuplements monospécifiques et réguliers, elles ne permettent pas de modéliser correctement les changements de structure et de composition de ces forêts. Au-delà de la première cohorte, le phénomène de remplacement des arbres fait en sorte que lâge rapporté par les inventaires forestiers et utilisé dans les tables de rendement sous-estime systématiquement lâge réel du peuplement (Kneeshaw et Gauthier 2003, Centre détude de la forêt 2008). De nombreuses études documentent la diversité et le dynamisme des vieilles forêts boréales (Franklin et al. 2002, Franklin et Van Pelt 2004, Gauthier et al. 2004, Gauthier 2009). Ladoption de courbes de rendement corrigées pourrait non seulement améliorer considérablement le calcul de la possibilité forestière (Garet 2009), mais également remettre en question l'idée que les vieilles forêts boréales sont sénescentes.

\section{Les vieilles forêts boréales sont plus susceptibles aux feux}

Les vieilles forêts boréales sont souvent perçues ou décrites comme étant plus susceptibles aux incendies forestiers, c'està-dire comme ayant plus de chance de brûler que les forêts plus jeunes. Les raisons évoquées pour appuyer ce mythe sont nombreuses. Laccumulation de débris ligneux au sol ou sur pied accroîtrait leur charge de combustible, les rendant ainsi plus susceptibles aux feux. La structure plus ouverte des vieilles forêts faciliterait leur assèchement, ce qui les rendrait plus susceptibles aux feux de forte intensité. Finalement, on associe à la structure étagée typique des vieux peuplements une plus grande facilité au développement de feux de couronne.

Or, les rares études qui portent sur lâge des peuplements et le risque de feu en forêt boréale suggèrent que le risque de feu est indépendant de lâge (p. ex., Bessie et Johnson 1995). Les résultats préliminaires de Leduc (2009) suggèrent même que les vieux peuplements dépinette noire seraient plus fréquemment représentés que les forêts plus jeunes dans les îlots épargnés par le feu à l’intérieur des brûlis.

\section{Les vieilles forêts boréales sont plus susceptibles aux épidémies d'insectes}

On considère souvent que laisser vieillir les peuplements au-delà de leur maturité commerciale augmente le risque dépidémie d'insectes à léchelle du paysage. Plusieurs études suggèrent toutefois que les vieilles forêts ne sont pas plus susceptibles aux épidémies de tordeuse de bourgeons de lépinette (TBE) que les forêts matures (MacLean 1980, MacLean et al. 2001, Alfaro et al. 2001). La susceptibilité aux épidémies de TBE, ou la probabilité d'infestation, semble atteindre un plateau au stade de maturité et naugmente plus par la suite. La relation entre la susceptibilité et lâge est donc sigmoïde : les peuplements immatures sont moins susceptibles que les peuplements matures et surannés, mais la susceptibilité ne continue pas d'augmenter avec lâge dans les peuplements qualifiés de surannés. 


\section{Les vieilles forêts boréales sont des sources de carbone}

On a longtemps considéré que les échanges de carbone entre les vieilles forêts boréales et l'atmosphère aboutissaient à un bilan neutre ou même que les vieilles forêts émettaient du carbone dans l'atmosphère (DeBell et Franklin 1987, Franklin et DeBell 1988, Waring et Running 1998). On supposait alors que l'émission de $\mathrm{CO}_{2}$ reliée à la respiration des décomposeurs et des plantes équivalait ou dépassait la consommation de $\mathrm{CO}_{2}$ réalisée lors de la photosynthèse.

Il est vrai de dire que la vitesse de séquestration du carbone par les vieilles forêts est plutôt faible. Des études récentes suggèrent par contre que le bilan de carbone des vieilles forêts est plus nuancé et plus dynamique quon ne le pensait (Suchanek et al. 2004) et que les vieilles forêts peuvent être un puits de carbone pendant des siècles (Luyssaert et al. 2008).

Il est faux de dire qu'un paysage composé de vieilles forêts perd du carbone. En effet, à l'échelle du paysage et en conditions naturelles, c'est le régime de perturbations qui dicte le niveau d'équilibre atteint entre les pertes et les gains de carbone. À cette échelle, le rajeunissement du paysage forestier par une intensification des perturbations, ou par l'introduction d'un nouveau type de perturbation telle que la coupe, entraîne nécessairement une baisse du stock de carbone avec le temps à léchelle du paysage jusquà l'atteinte d'un nouvel état. $\tilde{A}$ l'inverse, un ralentissement des perturbations engendre un vieillissement graduel du paysage forestier et une augmentation du stock de carbone moyen des peuplements.

\section{Conservation intégrale ou exploitation des vieilles forêts boréales}

Historiquement, l'enjeu de la conservation des vieilles forêts a été polarisé entre les groupes qui défendent les intérêts écologiques et patrimoniaux face aux groupes d'intérêts sociaux et économiques. Cependant, des positions beaucoup plus nuancées émergent lorsque les parties concernées expriment leurs intérêts propres lors de colloques ou d'ateliers. La certification forestière, par exemple, permet l'accès aux marchés tout en encourageant le maintien et l'aménagement durable des vieilles forêts. Les vieilles forêts boréales ont un bois qui possède des qualités recherchées, ce qui rend leur réduction en superficie équivalent à une décapitalisation du capital ligneux. Finalement, la diversité des vieilles forêts boréales peut être une ressource essentielle pour la diversification de léconomie locale des communautés forestières.

\section{II y a un clivage chercheurs-aménagistes concernant les vieilles forêts boréales}

La divergence de point de vue entre les chercheurs et les aménagistes existe bel et bien, mais elle a trop souvent été exagérée et les solutions pour y remédier ont trop souvent été négligées. Les aménagistes s'interrogent légitimement sur les coûts additionnels et la perte de productivité qui peuvent être associés à une stratégie visant le maintien des vieilles forêts boréales dans les territoires aménagés (Raedeke et al. 2001). Lopposition historique entre les intérêts économiques et les intérêts écologiques a favorisé un climat de méfiance nuisible à toute collaboration (Hillier 2003, Sarr et Puettmann 2008). En comprenant les objectifs et contraintes de l'industriel ainsi que les enjeux sociaux et écologiques, il est possible d'arriver à des solutions permettant à la fois de créer de la richesse tout en protégeant les fonctions écologiques des vieilles forêts et des forêts en général. Le clivage apparent entre les chercheurs et les aménagistes révèle en fait léchec du modèle traditionnel de transfert technologique utilisé ces dernières décennies et le manque de langage commun pour soutenir un véritable dialogue. La science documente de mieux en mieux les nombreuses fonctions écologiques associées aux vieilles forêts boréales, mais cette compréhension est rarement exprimée sous forme d'analyse économique. Labsence d'une vision plus globale n'a pas permis un synchronisme des préoccupations scientifiques, économiques et sociales concernant les vieilles forêts boréales et la forêt dans son ensemble.

\section{Des solutions adaptées à la conservation et à l'amé- nagement durable des vieilles forêts boréales}

Dans cette section, nous abordons plusieurs solutions identifiées lors de discussions entre divers intervenants du secteur forestier. Ces solutions témoignent de l'utilité et de l'efficacité du dialogue entre aménagistes et chercheurs, ainsi que de la nécessité d'inclure d'autres intervenants tels que les communautés locales, les économistes et les sociologues dans ces discussions. Lacceptabilité sociale de l'aménagement des vieilles forêts passe par une gestion prudente qui fait appel au principe de précaution (Bouthillier 2009). Plusieurs outils permettent d'améliorer cet aspect : l'utilisation de coupes à rétention variable, la protection d'une certaine proportion de vieilles forêts boréales et la certification, entre autres.

\section{Une stratégie formulée à différentes échelles spatiales et temporelles}

Notre approche actuelle de l'aménagement forestier considère le paysage forestier comme un assemblage de peuplements et omet de considérer les propriétés propres à cette échelle. Par exemple, alors qu'il est difficile de prédire quel peuplement brûlera dans la prochaine décennie, il est fort probable qu'une partie du paysage soit brûlée pendant cette même période. De la même manière, la protection intégrale d'une parcelle du paysage peut s'avérer inefficace si les forêts voisines sont fortement affectées par les perturbations naturelles ou anthropiques (Lindenmayer et al. 2008). La planification forestière devrait donc considérer l'impact cumulatif des perturbations sur le paysage forestier afin de pouvoir évaluer sa capacité d'atteindre ces objectifs, qu'ils soient économiques ou de conservation. Les objectifs d'aménagement doivent aussi être modulés selon léchelle de temps considérée, allant de volumes de récolte constants sur un horizon de quelques années à quelques décennies, jusqu'au maintien de services écologiques sur un horizon de plusieurs décennies ou siècles (Luckert et Williamson 2005, Bernier et al. 2007). Le maintien de vieilles forêts sous forme de massifs entre dans cette planification à long terme. La raréfaction des forêts âgées à l'échelle du paysage ainsi que la perte déléments structuraux caractéristiques des vieilles forêts à l'échelle du peuplement sont donc des enjeux qui mettent en évidence la nécessité de développer une véritable stratégie de gestion et d’aménagement des forêts boréales à différentes échelles spatiales et temporelles.

\section{Concilier aménagement et conservation des vieilles forêts boréales par une stratégie de zonage fonctionnel}

Le zonage fonctionnel (p. ex., Triade) est une stratégie d'aménagement où différents types d'utilisation du territoire sont 
distribués spatialement au sein d'une aire d’aménagement. Selon cette approche, les aires de conservation intégrale et d'aménagement intensif seraient réparties sur les sites les plus appropriés. Sur le reste du territoire, un aménagement extensif serait préconisé pour à la fois répondre aux besoins économiques (exploitation de ressources ligneuses et non ligneuses) et satisfaire les valeurs sociales (récréation, esthétisme, activités ancestrales, etc.) (Gauthier et al. 2008). Laménagement dit " extensif » pourrait regrouper un ensemble de stratégies d’aménagement de diverse intensité (en termes de prélèvement de matière ligneuse) qui pourrait viser plusieurs objectifs d'aménagement et de gestion, de façon intégrée. Par ailleurs, cette zone d’aménagement extensif pourrait agir comme une zone de transition en entourant les aires de conservation intégrale. Il ne sagit pas de mettre les vieilles forêts boréales seulement dans les aires de conservation, ni de conserver toutes les vieilles forêts, mais plutôt de s'assurer que les vieilles forêts soient bien représentées dans les aires de conservation selon leurs proportions dans le paysage régional. Par ailleurs, dans la portion aménagée, il faudrait maintenir des peuplements avec des caractéristiques de vieilles forêts en quantité suffisante pour constituer des corridors ou des « stepping stones » entre les zones de conservation.

\section{Des options sylvicoles adaptées aux vieilles forêts boréales}

Les options sylvicoles conventionnelles développées pour des peuplements forestiers de structure équienne ne permettent pas de créer ou de maintenir les structures et les fonctions associées aux vieilles forêts boréales. Par contre, certains de ces outils sylvicoles conventionnels, tels que la coupe sélective, peuvent être adaptés aux objectifs de maintien de certaines caractéristiques des vieilles forêts boréales (Ruel 2009). En effet, les recherches suggèrent que ce type de coupe permet de maintenir une structure résiduelle inéquienne et de réduire l'impact de l'aménagement forestier sur la biodiversité (Ruel 2009). Lapproche des trois cohortes (Bergeron et al. 1999, Bergeron 2009) va plus loin et place ces outils dans une perspective d'aménagement à léchelle du paysage. En effet, cette approche vise le maintien de forêts équiennes, irrégulières et très irrégulières telles que générées par un régime de perturbation naturelle par le biais d'interventions par coupe à faible rétention (coupe totale), par coupe partielle et par coupe sélective dans des proportions inspirées de celles caractérisant le paysage préindustriel.

Lutilisation de ces outils sylvicoles engendre toutefois une augmentation des coûts de récolte à court terme. Le déploiement de telles stratégies nécessite donc une évaluation plus poussée de leur rentabilité dans une perspective plus globale que les seuls coûts de récolte. De plus, il faut bien comprendre les réactions à long terme des structures résiduelles en termes de croissance et de mortalité, ainsi qu'en termes de maintien des attributs écologiques visés par la sylviculture.

\section{Diversifier l'économie locale des communautés forestières}

Plusieurs avantages économiques associés au maintien et à laménagement durable des vieilles forêts boréales ont été identifiés. Tout d'abord, la certification forestière est une approche permettant de donner une valeur économique à laménagement durable des vieilles forêts boréales et à leur maintien (conservation) dans les paysages aménagés. Les crédits de carbone potentiellement associés au maintien de massifs de vieilles forêts boréales pourraient également représenter un avantage économique relié au maintien des vieilles forêts boréales. Un tel marché existe déjà et permet dacheter des crédits de carbone en finançant des activités de plantation. Cependant, une telle approche ne serait pas efficace si elle conduisait à une substitution du bois par lacier et le béton dont la production émet davantage de $\mathrm{CO}_{2}$ que le bois.

Enfin, les vieilles forêts boréales intactes sont reconnues pour leur qualité esthétique, visuelle et patrimoniale. Des activités récréotouristiques pourraient également s'appuyer sur l'exploitation industrielle ou artisanale d'espèces forestières non ligneuses associées aux vieilles forêts boréales (plantes médicinales, baies, champignons, gibier) (Kneeshaw 2008). Ainsi, les vieilles forêts pourraient éventuellement contribuer à diversifier léconomie locale des communautés forestières. Cependant, en labsence d'une véritable analyse sociale et économique, cette perspective apparait encore anecdotique.

\section{Priorités de recherche pour améliorer notre compréhension des enjeux associés aux vieilles forêts boréales}

De nombreuses questions ralentissent la mise en place de scénarios sylvicoles adaptés aux vieilles forêts boréales et l'adoption d'un cadre de gestion qui permettrait d'agir dès maintenant dans le cadre des limites actuelles de nos connaissances.

\section{Définir des cibles d'aménagement et de conservation des vieilles forêts boréales à l'échelle du paysage}

De façon générale, en forêt boréale, le cycle de feu peut être utilisé pour calculer la proportion de vieilles forêts que les perturbations naturelles auraient maintenue en l'absence d’aménagement forestier (Bergeron et al. 1999). Les proportions de vieilles forêts protégées et aménagées à l'aide d’outils sylvicoles adaptés pourraient êtres basées sur la proportion de vieilles forêts calculée à l'aide du cycle de feu et de la longévité des espèces dominantes. On peut alors décider de maintenir une partie (p. ex., $50 \%$ ) ou la totalité de cette proportion dans les paysages soumis à un aménagement forestier industriel. Actuellement, seule la certification forestière exige l'établissement et le respect de cibles chiffrées en matière de vieilles forêts. La certification constitue donc un incitatif commercial pour le maintien d'une proportion de vieilles forêts équivalente à celle prévalant dans une forêt préindustrielle. Bien que le portrait des régimes de feu régionaux soit encore fragmentaire, plusieurs études documentent la fréquence des feux dans différentes régions du Québec boréal (Gauthier et al. 2008). La question actuellement débattue concerne les proportions relatives de vieilles forêts boréales à aménager et à conserver. Bien que la science nous permette aujourd'hui de calculer la proportion de vieilles forêts qui prévaudrait dans un paysage non aménagé, il revient ultimement à la société de choisir la proportion de vieilles forêts à maintenir dans un paysage soumis à l'exploitation industrielle afin de répondre aux attentes que la société nourrit envers les forêts et le secteur forestier. Ces valeurs sociales incluent des activités économiques viables et diversifiées à l'échelle locale, ainsi que les valeurs patrimoniales et culturelles que les populations 
expriment aujourd'hui par la voie des groupes environnementaux et des Premières nations, notamment.

Ces considérations rejoignent l'épineuse question du degré acceptable d'altération des vieilles forêts dans un paysage (Centre deétude de la forêt 2008). Bien que le système d'aménagement actuel nécessite des chiffres précis pour permettre le calcul de la possibilité forestière, il est risqué de s'appuyer sur des chiffres sans tenir compte de l'incertitude qui leur est associée, ni du caractère hautement dynamique des régimes de perturbations naturelles (Bonar et al. 2003). Il est prudent d'adopter des objectifs d'aménagement exprimés en termes de plage de variation (en se basant sur la variabilité historique, par exemple) et de pouvoir réviser périodiquement les objectifs d'aménagement au moyen d'une stratégie de gestion adaptative afin de tenir compte de lévolution de nos connaissances et du suivi de l'impact de nos interventions.

Mesurer l'impact de la fragmentation des massifs de vieilles forêts sur le maintien de la biodiversité et des processus écologiques

Il faudrait identifier les attributs-clés et les fonctions écologiques qui sont associées aux vieilles forêts boréales afin de développer des stratégies d'aménagement et de conservation adaptées. Nos connaissances actuelles sur la dynamique et la diversité de peuplements associés aux vieilles forêts boréales sont encore fragmentaires. Cependant, plusieurs études documentent la taille et la répartition des massifs forestiers intacts et perturbés à léchelle du paysage (Belleau et al. 2007, Belleau et Légaré 2008, Perron et al. 2008). En l'absence de perturbation anthropique, les brûlis sont séparés par de grandes distances et épargnent de grands massifs de vieilles forêts. La fragmentation de ces grands massifs forestiers par l'exploitation forestière crée des conditions nouvelles qui sortent parfois des limites de la variabilité naturelle auxquelles ces écosystèmes ont été assujettis. Dans la perspective de maintenir des écosystèmes sains et résilients qui fournissent des biens et services à la société, il faudrait mesurer l'impact de la foresterie sur les écosystèmes forestiers et leur dynamique à léchelle du paysage afin que les gestionnaires établissent des seuils d'altération acceptables en termes de proportion et de répartition des massifs de vieilles forêts. Par ailleurs, des mesures d'atténuation pourront être développées comme le maintien de corridors ou de "stepping stones " pour assurer les échanges et suivre les impacts sur les espèces clés et les processus écologiques.

Investir en recherche et innovation pour développer de nouveaux produits forestiers ligneux et non ligneux

Il faudrait penser à retirer de ce type de forêt des produits à haute valeur ajoutée dans la perspective de conserver et d'aménager durablement les vieilles forêts. La certification environnementale a déjà permis de donner une valeur accrue aux produits forestiers courants. Cependant, la diminution de la demande en pâte et papier incite à développer de nouveaux produits et de nouveaux marchés. Par exemple, le bois provenant des arbres matures des vieilles forêts possède des propriétés particulières et pourrait donc préférentiellement servir aux bois d'ingénierie. Les entreprises qui parviennent à tirer leur épingle du jeu dans le contexte de la crise forestière actuelle sont celles qui développent des produits innovateurs (Beauregard 2009). De plus, si l’on pense à diversifier les produits ligneux retirés de nos forêts depuis quelques décennies, l'exploitation des produits forestiers non-ligneux est encore embryonnaire au Québec et devrait faire l'objet de recherches spécifiques (Duchesne et Wetzel 2003).

\section{Développer notre expertise en économie forestière}

La principale réticence envers l'aménagement écosystémique est le manque d'analyse économique : combien va coûter la transition d'un système d'aménagement équienne à un aménagement écosystémique? Comment intégrer les pratiques d'aménagement écosystémique dans le calcul de la possibilité forestière? Y aura-t-il des pertes d'opportunités si une option est choisie plutôt qu'une autre? Il est nécessaire de faire l'analyse économique d'un changement de paradigme en aménagement forestier tel que la transition d'une stratégie d'aménagement à rendement soutenu à une stratégie d'aménagement écosystémique. Par ailleurs, les concepts permettant de gérer les risques financiers, tels que la théorie des options réelles, les approches d'optimisation, les approches de précaution et les approches robustes, sont de plus en plus appliqués à la gestion des ressources naturelles parce que de nombreux paramètres sont incertains et changeants dans le temps (Lempert et Collins 2007, Morgan et al. 2008).

\section{Analyser les nouvelles valeurs sociales des forêts et développer de nouveaux outils de gestion}

L'analyse économique du secteur forestier devrait être réalisée à la lumière des attentes sociales que nous avons par rapport aux forêts : souhaitons-nous des emplois, de la ressource ligneuse, ou encore des forêts accessibles à des activités récréotouristiques? Ou bien tout cela à la fois? Dans tous les cas, pour susciter une acceptabilité sociale accrue, le processus de gestion forestière devrait être plus transparent et plus inclusif (Bouthillier 2009). C'est pourquoi il faudrait développer les moyens nécessaires pour mettre en place une stratégie de gestion qui soit davantage participative. Plusieurs approches sylvicoles et d'aménagement forestier durable permettent de concilier les objectifs d'aménagement et de conservation des vieilles forêts boréales et de leurs attributs en s'appuyant sur notre compréhension des processus écologiques sous-jacents à la dynamique naturelle. Cependant, les vieilles forêts boréales soulèvent plusieurs enjeux économiques et sociaux qui devront eux aussi faire lobjet de recherches plus poussées.

\section{Conclusion}

Les vieilles forêts boréales cristallisent plusieurs problématiques forestières comme l'aménagement forestier durable et le développement d'un réseau d'aires protégées, mais aussi la gestion adaptative et participative des ressources naturelles. Ainsi, les vieilles forêts boréales offrent une bonne occasion pour l'industrie forestière de développer une gestion transparente qui intègre l'ensemble des valeurs sociales associées aux vieilles forêts. Nos pratiques sylvicoles conventionnelles sont mal adaptées à la structure inéquienne des vieilles forêts boréales et aux grands massifs qu'elles forment naturellement. Leur diversité pourrait contribuer au développement de solutions pour faire face à la crise actuelle. Cependant, une véritable concertation entre les industriels, les communautés locales, le public, le gouvernement et les chercheurs est nécessaire pour y parvenir. Par ailleurs, le cadre actuel de gestion 
des forêts, qui est très normatif, ne permet pas d’ajuster les objectifs et les approches d’aménagement pour mieux refléter notre compréhension changeante des vieilles forêts boréales.

\section{Remerciements}

Nous remercions tous les participants et présentateurs qui ont contribué au colloque sur les vieilles forêts boréales, qui a eu lieu à Sept-Îles du 27 au 29 mai 2008, pour avoir stimulé les réflexions qui ressortent dans cet article. Les commentaires de Sylvie Gauthier nous ont permis d’améliorer la qualité du manuscrit. Nous remercions également Isabelle Lamarre pour lédition de manuscrit.

\section{Références}

Alfaro, R.I., S. Taylor, R.G. Brown et J.S. Clowater. 2001. Susceptibility of northern British Columbia forests to spruce budworm defoliation. For. Ecol. Manag. 145: 181-190.

Beauregard, R. 2009. Particularité du bois des vieilles forêts, usages industriels et discussion des modèles d'affaire. Dans Actes du colloque « Les vieilles forêts boréales : leur place dans l'aménagement forestier » tenu les 28 et 29 mai 2008 à Sept-Îles, Québec. pp. 65-66. Belleau, A., Y. Bergeron, A. Leduc, S. Gauthier et A. Fall. 2007. Using spatially explicit simulations to explore size distribution and spacing of regenerating areas produced by wildfire: recommendations for designing harvest agglomerations for the Canadian boreal forest. For. Chron. 83: 72-83.

Belleau, A. et S. Légaré. 2008. Projet Tembec : vers la mise en oeuvre d'une stratégie d'aménagement forestier s'inspirant de la dynamique des perturbations naturelles pour la région nord de l'Abitibi. Dans S. Gauthier, M.-A. Vaillancourt, A. Leduc, L. De Grandpré, D. Kneeshaw, H. Morin, P. Drapeau et Y. Bergeron (dir.). Aménagement écosystémique en forêt boréale. pp. 507-528. Presses de l'Université du Québec, Québec, QC.

Bergeron, Y. 2009. Comment concilier récolte et présence de forêts de structure ancienne sur de grands territoires : le concept des trois cohortes est-il applicable? Dans Actes du colloque « Les vieilles forêts boréales : leur place dans l'aménagement forestier » tenu les 28 et 29 mai 2008 à Sept-Îles, Québec. pp. 40-47.

Bergeron, Y., B. Harvey, A. Leduc et S. Gauthier. 1999. Forest management guidelines based on natural disturbance dynamics: stand- and forest-level considerations. For. Chron. 75: 49-54.

Bernier, P., A. Leduc et F. Raulier. 2007. Repenser le rendement soutenu dans la foresterie québécoise. L'Aubelle 152: 11-12.

Bessie, W.C. et E.A. Johnson. 1995. The relative importance of fuels and weather on fire behavior in subalpine forests. Ecology 76: 747-762.

Bonar, R.L., H. Lougheed et D.W. Andison. 2003. Natural disturbance and old-forest management in the Alberta Foothills. For. Chron. 79: 455-461.

Boudreault, C. 2001. Facteurs-clés pour le maintien de la diversité des lichens épiphytes. Nat. Can. 125: 175-179.

Bouthillier, L. 2009. Acceptabilité sociale et gestion des vieilles forêts boréale. Dans Actes du colloque « Les vieilles forêts boréales : leur place dans l'aménagement forestier » tenu les 28 et 29 mai 2008 à Sept-Îles, Québec. pp. 33-39.

Bouthillier, L. et A. Roberge. 2007. Les intentions des programmes de participation du public appliqués par l'industrie forestière : état de la situation au Québec. For. Chron. 83: 810-817.

Centre d'étude de la forêt. 2008. Avis scientifique portant sur l'article 92.0.3.2 de la Loi sur les forêts. Disponible en ligne : http://www.forestierenchef.gouv.qc.ca/fichiers/documents/contenu/Aviscef.pdf [dernière consultation le 10 mars 2009].

Courtois, R., J.-P. Ouellet, A. Gingras, C. Dussault et D. Banville. 2001. La situation du caribou forestier au Québec. Nat. Can. 125: 53-63.
DeBell, D.S. et J.F. Franklin. 1987. Old-growth Douglas-fir and western hemlock: a 36-year record of growth and mortality. West. J. Appl. For. 2: 111-114.

De Grandpré, L., S. Gauthier, C. Allain, D. Cyr, S. Périgon, A.T. Pham, D. Boucher, J. Morissette, G. Reyes, T. Aakala et T. Kuuluvainen. 2008. Vers un aménagement écosystémique de la forêt boréale de la Côte-Nord : régime des perturbations et dynamique naturelle. Dans S. Gauthier, M.-A. Vaillancourt, A. Leduc, L. De Grandpré, D. Kneeshaw, H. Morin, P. Drapeau et Y. Bergeron (dir.). Aménagement écosystémique en forêt boréale. pp. 241-268. Presses de l'Université du Québec, Québec, QC.

Duchesne, L.C. et S. Wetzel. 2003. Laménagement des produits forestiers non ligneux et des ressources ligneuses des forêts canadiennes : besoins d'intégration et de recherche. For. Chron. 79: 853-859.

Euler, D. et C. Wedeles. 2005. Defining old-growth in Canada and identifying wildlife habitat in old-growth boreal forest stands. National Council for Air and Stream Improvement, Inc. Technical Bulletin No. 0909. Research Triangle Park, NC. Disponible en ligne : http://www.ncasi.org//Publications/Detail.aspx?id=2832. [Dernière consultation le 12 août 2009].

Franklin, J.F. et D.S. DeBell. 1988. Thirty-six years of tree population change in an old-growth Pseudotsuga-Tsuga forest. Rev. Can. Rech. For. 18: 633-639.

Franklin, J.F, T.A. Spies, R. Van Pelt, A.B. Carey, D.A. Thornburgh, D.R. Berg, D.B. Lindenmayer, M.E. Harmon, W.S. Keeton, D.C. Shaw, K. Bible et J. Chen. 2002. Disturbances and structural development of natural forest ecosystems with silvicultural implications, using Douglas-fir forests as an example. For. Ecol. Manage. 155: 399-423.

Franklin, J.F. et R. Van Pelt. 2004. Spatial aspects of structural complexity in old-growth forests. J. For. 102(3): 22-28.

Garet, J. 2009. Rendement des vieilles forêts d'épinette noire. Dans Actes du colloque "Les vieilles forêts boréales : leur place dans l'aménagement forestier » tenu les 28 et 29 mai 2008 à Sept-Îles, Québec. pp. 53-59.

Gauthier, S. 2009. Dynamique des vieilles forêts, leur évolution en matière de structure et de composition en fonction des perturbations qui y sévissent. Dans Actes du colloque "Les vieilles forêts boréales : leur place dans l’aménagement forestier » tenu les 28 et 29 mai 2008 à Sept-Îles, Québec. pp. 14-18.

Gauthier, S., J. Morissette, D. Boucher et L. De Grandpré. 2004. Succession forestière dans la forêt boréale de la Côte-Nord du Québec : Facteurs impliqués dans les changements de composition des espèces sur une période de près de 60 ans. Dans Amélioration de la précision du calcul de la possibilité forestière par une meilleure connaissance de la dynamique naturelle de la forêt boréale de la Côte-Nord. Rapport final présenté au Fonds forestier, Direction de la recherche forestière, ministère des Ressources naturelles, de la Faune et des Parcs. Projet $n^{\circ} 03123125$.

Gauthier, S., A. Leduc, Y. Bergeron, et H. Le Goff. 2008. La fréquence des feux et l'aménagement forestier inspiré des perturbations naturelles. Dans S. Gauthier, M.-A. Vaillancourt, A. Leduc, L. De Grandpré, D. Kneeshaw, H. Morin, P. Drapeau et Y. Bergeron (dir.). Aménagement écosystémique en forêt boréale. pp. 61-78. Presses de l'Université du Québec, Québec, QC.

Hillier, J. 2003. Fighting over the forests: Environmental conflict and decision-making capacity in forest planning processes. Aust. Geogr. Stud. 41: 251-269.

Jetté, J.-P., M.-A. Vaillancourt, A. Leduc, et S. Gauthier. 2008. Les enjeux écologiques de l'aménagement forestier. Dans S. Gauthier, M.-A. Vaillancourt, A. Leduc, L. De Grandpré, D. Kneeshaw, H. Morin, P. Drapeau et Y. Bergeron (dir.). Aménagement écosystémique en forêt boréale. pp. 1-10. Presses de l'Université du Québec, Québec, QC. 
Kneeshaw, D. 2008. L'écotourisme dans les territoires forestiers : Une source de conflit ou une possibilité de meilleure gestion? Dans M. Lequin et B. Sarrazin (dir.) Tourisme et territoires forestiers Vers de nouvelles perspectives de mise en valeur. pp 233-246. Presses de l'Université du Québec. Montréal, QC.

Kneeshaw, D. et S. Gauthier. 2003. Old growth in the boreal forest: A dynamic perspective at the stand and landscape level. Environ. Rev. 11: S99-S114.

Leduc, A. 2009. Spécificité des feux envers lâge des peuplements : les vieilles forêts sont-elles des «niques » à feu? Dans Actes du colloque « Les vieilles forêts boréales : leur place dans l’aménagement forestier " tenu les 28 et 29 mai 2008 à Sept-Îles, Québec. pp. 19-23. Lempert, R.J. et M.T. Collins. 2007. Managing the risk of uncertain threshold responses: comparison of robust, optimum, and precautionary approaches. Risk Anal. 27: 1009-1026.

Lindenmayer, D. et al. 2008. A checklist for ecological management of landscapes for conservation. Ecol. Lett. 11: 78-91.

Luckert, M.K. et T. Williamson. 2005. Should sustained yield be part of sustainable forest management? Rev. Can. Rech. For. 35: $356-364$

Luyssaert, S.E., E.D. Schulze, A. Börner, A. Knohl, D. Hessenmöller, B.E. Law, P. Ciais et J. Grace. 2008. Old-growth forests as global carbon sinks. Nature 455: 213-215.

McCarthy, J. 2001. Gap dynamics of forest trees: a review with particular attention to boreal forests. Environ. Rev. 9: 1-59.

MacLean, D.A. 1980. Vulnerability of fir-spruce stands during uncontrolled spruce budworm outbreaks: a review and discussion. For. Chron. 56: 213-221.

MacLean, D.A., T.A. Erdle, W.E. MacKinnon, K.B. Porter, K.P. Beaton, G. Cormier, S. Morehouse et M. Budd. 2001. The Spruce Budworm Decision Support System: forest protection planning to sustain long-term wood supply. Rev. Can. Rech. For. 31: 1742-1757.
Morgan, D.G., S. Ben Abdallah et P. Lasserre. 2008. A real options approach to forest-management decision making to protect caribou under the threat of extinction. Ecol. Soc. 13: 27. Disponible en ligne : http://www.ecologyandsociety.org/vol13/iss1/art27/ [dernière consultation le 20 janvier 2009].

Perron, N., L. Bélanger et M.-A. Vaillancourt. 2008. Organisation spatiale des peuplements et de la forêt résiduelle sous régimes de feu et de coupes. Dans S. Gauthier, M.-A. Vaillancourt, A. Leduc, L. De Grandpré, D. Kneeshaw, H. Morin, P. Drapeau et Y. Bergeron (dir.). Aménagement écosystémique en forêt boréale. pp. 137-164. Presses de l'Université du Québec, Québec, QC.

Pham, A.T., L. De Grandpré, S. Gauthier et Y. Bergeron. 2004. Gap dynamics and replacement patterns in gaps of the northeastern boreal forest of Quebec. Rev. Can. Rech. For. 34: 353-364.

Pothier, D. et F. Savard. 1998. Actualisation des tables de production pour les principales espèces forestières du Québec. Ministère des Ressources naturelles du Québec, Forêt Québec. 183 p.

Raedeke, A.H., J.S. Rikoon et C.H. Nilon. 2001. Ecosystem management and landowner concern about regulations: A case study in the Missouri Ozarks. Soc. Nat. Res. 14: 741-759.

Ruel, J.-C. 2009. Options sylvicoles applicables aux vieilles forêts. Dans Actes du colloque "Les vieilles forêts boréales : leur place dans l'aménagement forestier " tenu les 28 et 29 mai 2008 à SeptÎles, Québec. pp. 60-64.

Sarr, D.A. et K.J. Puettmann. 2008. Forest management, restoration, and designer ecosystems: Integrating strategies for a crowded planet. Écoscience 15: 17-26.

Suchanek, T.H., H.A. Mooney, J.F. Franklin, H. Gucinski et S.L. Ustin. 2004. Carbon dynamics of an old-growth forest. Ecosystems 7: 421-426.

Waring, R.H. et S.W. Running. 1998. Forest Ecosystems: Analysis at Multiple Scales. 2nd Edition. Academic Press, Toronto, ON. 